\title{
Reflectometria no domínio do tempo na determinação do conteúdo de água no solo
}

\author{
Sidney Pereira ${ }^{1}$, Delly Oliveira Filho ${ }^{2}$, Everardo C. Mantovani ${ }^{2}$, Márcio M. Ramos ${ }^{2} \&$ José H. Martins ${ }^{2}$
}

\begin{abstract}
RESUMO
Teve-se como objetivo principal, neste estudo, determinar a correlação existente entre a constante dielétrica aparente e o conteúdo de água para um solo de textura argilosa e outro de textura arenosa, por meio de ajuste de modelo do tipo polinômio cúbico. Propõe-se um sistema TDR para medição da umidade do solo, formado por: osciloscópio; gerador de pulsos eletromagnéticos; cabo coaxial de $50 \Omega$; cabo de antena de TV; casador de impedâncias; sondas (hastes metálicas paralelas) com comprimentos de 0,10,0,15,0,20,0,25, 0,30 e 0,35 m, com dois diâmetros de hastes $\left(\mathrm{d}_{1}=3,2\right.$ e $\mathrm{d}_{2}=6,5 \mathrm{~mm}$ ). Para o solo arenoso, o melhor ajuste ocorreu para a sonda de $0,30 \mathrm{~m}$ de comprimento e diâmetro de hastes de 3,2 mm, obtendo-se um coeficiente de determinação ajustado, $\mathrm{R}^{2}$, de 0,954 , enquanto para o solo argiloso 0 melhor ajuste se verificou para a sonda de 0,30 m de comprimento, com diâmetro de hastes de 3,2 mm, obtendo-se um coeficiente de determinação ajustado $R^{2}$ de 0,923 . Enfim, o sistema TDR proposto estimou a umidade do solo argiloso com erro médio relativo de $6,6 \%$, para sonda de $0,30 \mathrm{~m}$.
\end{abstract}

Palavras-chave: TDR, sonda, automação e irrigação

\section{Time domain reflectometry in the determination of soil water content}

\begin{abstract}
This study had as objective the determination of the correlation between the apparent dielectric constant and water content for a clay-textured soil and a sandy-textured soil by fitting a cubic polynomial type model. To measure the soil water content a TDR system was proposed, which consists of: oscilloscope; electromagnetic pulse generator; coaxial cable of $50 \Omega$; TV antenna cable; impedance matching; probes (paralleled metallic guides) with $0.10,0.15,0.20,0.25,0.30$ and $0.35 \mathrm{~m}$ lengths and with two diameters $\left(\mathrm{d}_{1}=3.2\right.$ and $\left.\mathrm{d}_{2}=6.5 \mathrm{~mm}\right)$. For the sandy soil, the best fit was obtained with a 0.30 m-long probe with $3.2 \mathrm{~mm}$-diameter guides, an adjusted determination coefficient, $\mathrm{R}^{2}$, of 0.954 . For the clay soil, the best fit was obtained with a $0.30 \mathrm{~m}$-long probe with a $3.2 \mathrm{~mm}$-diameter guides, and an adjusted determination coefficient, $\mathrm{R}^{2}$, of 0.923 . The TDR system, proposed for determination of the soil water content within the range between the field capacity and the wilting point, provided the best determination coefficients for the $0.30 \mathrm{~m}$ probe in both soils and the studied probe lengths. The proposed TDR system estimated the clay-soil water content with mean relative error of $6.6 \%$, for the $0.30 \mathrm{~m}$ probe.
\end{abstract}

Key words: TDR, probe, irrigation and automation 


\section{INTRODUÇÃO}

Com a crise de fornecimento de energia elétrica por que atravessou o País, racionamento de energia de 2001, o uso deste recurso e da água destinada à irrigação se torna cada vez mais restritivo e oneroso, implicando em prioridade para sua otimização em seus diferentes usos, tanto no contexto estratégico de reservas energéticas quanto sob o ponto de vista ecológico. Em razão disto, técnicas devem ser desenvolvidas e aplicadas em sistemas de irrigação, para que os processos agrícolas se tornem mais eficientes na utilização desses recursos, em conseqüência dos produtores competitivos. Assim, a determinação da umidade em solos destinados à produção agrícola é de vital importância para o manejo adequado da água e, conseqüentemente, da energia para a pressurização dos sistemas de irrigação; por sua vez, o manejo eficiente desses sistemas favorece o desenvolvimento adequado das plantas e contribui para o aumento da produção e preservação do meio ambiente. No Brasil, em sua maioria os usuários de grandes áreas irrigadas não fazem o manejo adequado da água e o monitoramento automático é feito quase que exclusivamente no âmbito dos centros de pesquisa.

A técnica TDR passou a ser utilizada como meio para medição da constante dielétrica em líquidos, no fim da década de 60, por Feellner-Fedegg, citado por Gomide (1998); desde então, esta técnica passou a ser usada para medição da constante dielétrica em diversos materiais. No início da década de 80 ela foi empregada para determinação de propriedades físicas do solo, quando se verificou que a constante dielétrica possuía relação acentuada com o teor de água e, em menor grau, com a composição e densidade do mesmo. Diversos trabalhos foram desenvolvidos para medição do teor de umidade do solo por meio da determinação da constante dielétrica, com os equipamentos testadores de cabos elétricos associados a outros equipamentos eletrônicos.

A determinação do conteúdo de água do solo é obtida a partir da estimativa da constante dielétrica calculada, usando-se a técnica da reflectometria no domínio do tempo; este valor é baseado no tempo de emissão/reflexão de um pulso eletromagnético, emitido por um gerador de pulsos, em hastes metálicas paralelas ou não, que servem como guia de ondas. A constante dielétrica determinada será a do material entre as hastes metálicas, i.e. a do solo. A configuração da sonda com suas hastes assemelha-se à construção de um capacitor, em que o material dielétrico é o próprio solo. A partir desta analogia pode-se demonstrar, resumidamente, como se obtém o valor da constante dielétrica.

Diversos são os modelos para a calibração do TDR, sendo o proposto por Topp et al. (1980) o que tem sido usado como referência. Este modelo admite que a constante dielétrica aparente do solo seria apenas uma função do conteúdo de água do mesmo. O modelo desconsidera as demais variáveis do solo mas apresenta melhores coeficientes de ajuste em solos homogêneos, sendo utilizado por diversos fabricantes de equipamentos TDR, a fim de medir o conteúdo de água do solo, uma vez que apresenta coeficientes de ajuste elevados em solos minerais com conteúdo de água menor que $0,5 \mathrm{~cm}^{3} \mathrm{~cm}^{-3}$ (Serrarens, et al., 2000).
A grande dificuldade no uso da técnica de reflectometria no domínio do tempo, para a medição da umidade do solo, é sua calibração. Atualmente, não há um modelo admitido como padrão de calibração mas existem, sim, pesquisas que visam sistematizar essa calibração (White et al., 1994). Para freqüências entre 1 e $20 \mathrm{~Hz}$, a variável que afeta preponderantemente a constante dielétrica aparente do solo seria o conteúdo de água no mesmo, Davis e Annan, citados por Nissen \& Moldrup (1998) e Amato \& Ricthie (1995). Outras variáveis que afetam secundariamente a determinação da constante dielétrica são, textura, densidade, estrutura, teor de sais solúveis, temperatura e freqüência da onda eletromagnética utilizada Topp et al. (1980).

A dificuldade de se encontrar um modelo padrão consiste, entre outros fatores, na composição atípica de alguns solos. Dados obtidos por meio de calibrações feitas em laboratórios, podem ser encontrados na literatura (Dirksen \& Dasberg, 1993). Roth et al. (1992) mostraram que são necessárias calibrações diferentes para solos com minerais ferrosos ou orgânicos. Segundo Robinson et al. (1994), a presença de óxido de ferro e minerais de óxido de titânio influencia a determinação da constante dielétrica por meio da tecnologia TDR e a subseqüente estimação do conteúdo de água.

Através deste trabalho objetivou-se, sobretudo, desenvolver um sistema para medição da umidade do solo, por meio da determinação de sua constante dielétrica, utilizando-se o princípio da reflectometria no domínio do tempo (TDR). Os objetivos específicos, foram: (i) determinar a correlação existente entre a constante dielétrica e o conteúdo de água para solos argilosos e arenosos, e (ii) determinar o diâmetro e o comprimento de sondas com melhor desempenho para utilização com o TDR.

\section{MATERIAL E MÉTODOS}

O trabalho foi realizado nos Laboratórios de Instrumentação e de Hidráulica do Departamento de Engenharia Agrícola da Universidade Federal de Viçosa, Viçosa, Minas Gerais. O equipamento TDR montado para medição da umidade do solo, se compunha de: osciloscópio de $500 \mathrm{~Hz}$ (modelo HP 54610B, HP), gerador de pulsos (modelo 12562/D, EMG), cabo coaxial, cabo de antena para TV, casador de impedâncias, conectores e sondas, conforme ilustrado na Figura 1.

O osciloscópio utilizado possui interface de comunicação, permitindo que os sinais detectados pelo aparelho sejam enviados para o computador. Um software específico foi desenvolvido para converter cada sinal (ou forma de onda) visualizado na tela do osciloscópio em 2.000 pares ordenados, que foram armazenados em uma planilha eletrônica, para posterior análise. A forma de onda quadrada empregada foi a padrão, utilizada neste estudo com 5,844 volts pico a pico, freqüência de $2 \mathrm{~Hz}$ e uma subida de pico em aproximadamente 5,6 ns e impedância de saída de $50 \Omega$.

O cabo coaxial de $50 \Omega$ foi utilizado para fazer a conexão entre o osciloscópio e o gerador de pulsos; ele foi selecionado 


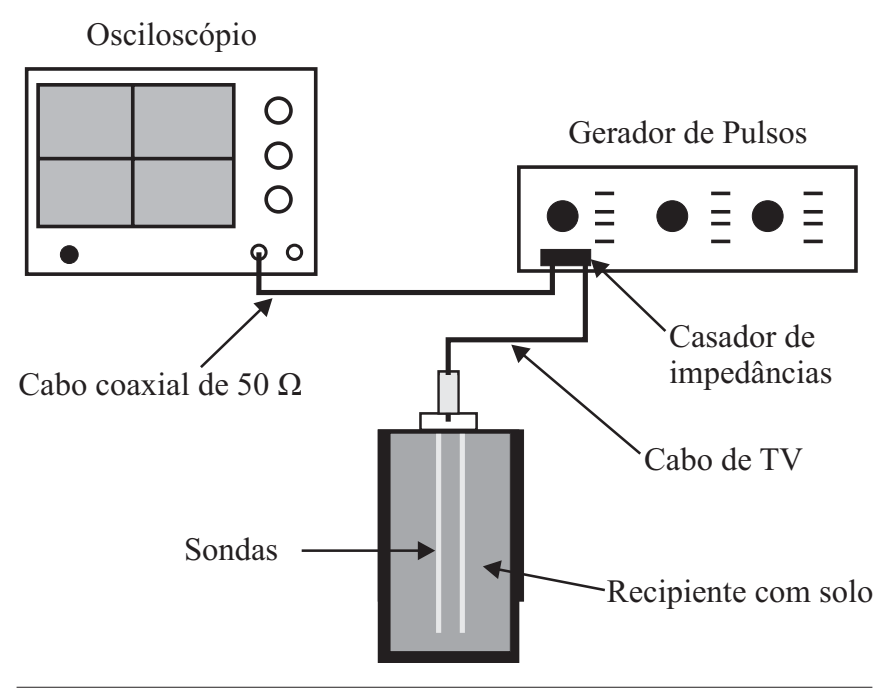

$\overline{\text { Figura 1. Sistema para medição da umidade do solo utilizando-se o }}$ princípio da reflectometria no domínio do tempo

por apresentar menor influência nas reflexões do sinal. O cabo de conexão utilizado neste trabalho foi o de antena de TV, paralelo, de $300 \Omega$, com cerca de $1 \mathrm{~m}$ de comprimento, o qual, embora possuindo impedância maior que o cabo coaxial de $50 \Omega$, apresentou-se como o mais viável, tecnicamente, para fazer a conexão das sondas ao casador de impedâncias; este, por sua vez, era conectado ao gerador de pulsos por meio de uma derivação em T; o outro terminal da derivação $\mathrm{T}$ foi conectado ao osciloscópio.

O casador de impedâncias usado foi do tipo 1:1. Ressalta-se que a magnitude e o sinal da reflexão sofrida em sondas TDR, para medição da umidade do solo, dependem da magnitude do casador de impedâncias; portanto, casadores 1:1 ou 1:4 teriam diferentes reflexões para uma combinação de cabos e sondas.

A impedância z para linhas de transmissão paralelas (sondas) em um meio preponderantemente não-condutivo (solo) é função de constante dielétrica ka e foi calculada pela Eq. 1, segundo Grant \& Philips, citados por Spaans \& Baker (1993), Huisman \& Bouten (1999), Noborio et al. (1999), válida para $2 \mathrm{~s}>>$ d, ou

$$
z=\frac{120}{\sqrt{\mathrm{ka}}} \ln \left(\frac{2 \mathrm{~s}}{\mathrm{~d}}\right)
$$

em que

Z - impedância da sonda, $\Omega$

$\mathrm{S}$ - espaçamento entre as sondas, $\mathrm{m}$

d - diâmetro das sondas, $m$

Foram construídos dois conjuntos de sondas TDR, das quais seis com diâmetro $d_{1}$ de $3,2 \mathrm{~mm}$ e outras seis com diâmetro $\mathrm{d}_{2}$ de $6,5 \mathrm{~mm}$. Cada sonda era composta de duas hastes paralelas, espaçadas entre si $50 \mathrm{~mm}$. Construíramse, também, sondas com diâmetros $\mathrm{d}_{1}$ e $\mathrm{d}_{2}$ e comprimentos de 0,$10 ; 0,15 ; 0,20 ; 0,25 ; 0,30$ e $0,35 \mathrm{~m}$. As sondas TDR, para medição da umidade do solo, foram feitas com material não-magnético e condutividade elétrica muito maior que a do meio em que foram inseridas (Dalton, 1992). Neste experimento, os fios do cabo de TV foram soldados a dois conectores do tipo "pino banana” e, em cada haste da sonda, foi atarraxado, nas extremidades que não ficavam enterradas no solo, um conector tipo "sindal”; na outra extremidade deste último conector aparafusou-se um fio rígido, com cerca de $20 \mathrm{~mm}$ na extremidade do qual, foi soldado um outro “pino banana”, que facilmente poderia ser conectado e desconectado ao cabo de TV. Este procedimento foi necessário, uma vez que um grande número de acoplamentos seria feito durante o experimento, além de e também para evitar reflexões desnecessárias que poderiam ocorrer em conexões mais simples.

As hastes foram mantidas paralelas entre si, condição essencial para medição da condutividade elétrica do meio; para tal, utilizaram-se dos segmentos de tubos hidráulicos de PVC, com 12 e 19 mm de diâmetro, para as sondas com os diâmetros $d_{1}$ e $d_{2}$, respectivamente, Figura 1. Os segmentos de PVC com $70 \mathrm{~mm}$ de comprimento foram preenchidos com massa epóxi, para conferir rigidez ao conjunto.

\section{Solos ensaiados}

Os solos estudados foram coletados nos municípios de Coimbra e São Geraldo, Minas Gerais, selecionando-se dois solos homogêneos: Areia Quartzosa (AQ) e Latossolo Vermelho (LV), com características granulométricas bem distintas. Essas análises foram realizadas no Laboratório de Solos do Departamento de Fitotecnia da Universidade Federal de Viçosa. A curva de retenção de água no solo foi determinada com o extrator de pressão de Richards, utilizando-se amostras deformadas. Os pontos de interesse da curva de retenção, para este estudo, se concentram entre as umidades correspondentes ao ponto de murcha permanente e a de capacidade de campo dos solos.

\section{Homogeneização da umidade dos solos}

Seguiu-se a metodologia de Tommaselli (1997), para se efetuar a medição da umidade do solo, ou seja, determinaram-se no mínimo vinte valores da constante dielétrica e as respectivas umidades do solo, distribuídos uniformemente entre o ponto de umidade residual e o ponto de saturação do solo, durante o delineamento da curva de calibração da tecnologia TDR. Para se obter esses pontos uniformemente distribuídos, adotou-se a seguinte metodologia. Foram secados ao ar, destorroados e peneirados em malhas de $2 \mathrm{~mm}$; após serem peneirados, as amostras foram armazenadas em recipientes construídos especialmente para este fim, cujos recipientes foram constituídos de segmentos de tubos de PVC de $200 \mathrm{~mm}$ de diâmetro externo e altura de $0,40 \mathrm{~m}$ e a base foi concretada com uma argamassa de traço forte, com $30 \mathrm{~mm}$ de altura, para que não se perdesse água pela base do recipiente. A altura útil de cada recipiente era de $0,37 \mathrm{~m}$ de camada de solo.

Antes de fazer o preenchimento dos recipientes, determinou-se a umidade residual, pelo método padrão de estufa. O intervalo entre a umidade residual e a capacidade de campo foi dividido em 20 partes, obtendo-se a umidade que cada solo contido no recipiente deveria possuir. Subtraindo-se da umidade desejada o valor da umidade residual, calculou-se a quantidade de água a ser adicionada ao solo 
de cada recipiente, multiplicando-se a diferença pelo peso do solo. A massa de água foi cuidadosamente aspergida sobre a massa de solo e logo após se procedeu à mistura do solo umedecido, peneirada duas vezes consecutivas, em malhas de $2 \mathrm{~mm}$ e, finalmente, depositada de volta no recipiente. Após a deposição do solo, o recipiente foi vedado com plástico e cinta elástica e deixado em repouso por, no mínimo, 10 dias para que houvesse a homogeneização da umidade em todo o perfil.

Para determinação da umidade de saturação dos solos utilizaram-se recipientes de PVC com 200 mm de diâmetro externo, com base de PVC perfurada e com uma malha porosa em sua parte interna, disposta no fundo do recipiente. Foram usados dois recipientes imersos em água, até uma altura da coluna correspondente a $2 / 3$ de solo, durante três dias, antes da aquisição de dados. Determinou-se, também, a constante dielétrica dos dois tipos de solo secado em estufa com 105 a $110^{\circ} \mathrm{C}$, por $72 \mathrm{~h}$. Antes da aquisição de dados esperou-se, durante $6 \mathrm{~h}$, que o solo atingisse temperatura ambiente.

\section{Determinação da umidade volumétrica do solo}

Para o cálculo do conteúdo volumétrico de água do solo contido nos recipientes após a aquisição de dados com o sistema TDR desenvolvido, determinou-se a densidade do solo úmido $\rho_{\mathrm{u}}$ e a umidade gravimétrica $\mathrm{U}_{\mathrm{g}}$ de cada amostra. A densidade do solo úmido foi determinada pela razão entre a massa do solo úmido e o volume do solo no recipiente.

Para determinação da umidade gravimétrica do solo foram coletadas quatro amostras de cada recipiente, nas profundidades de 0,05, 0,10, 0,20 e 0,30 m e, para a determinação da umidade média, utilizou-se o TDR, procedendo-se da seguinte forma: (i) para sondas de 0,10 e $0,15 \mathrm{~m}$, usouse a média dos valores de umidade gravimétrica obtidos a 5 e $10 \mathrm{~cm}$ de profundidade; (ii) para sondas de 0,20 e 0,25 m, lançou-se mão da média dos valores de umidade gravimétrica obtidos a 0,05; 0,10 e 0,20 m de profundidade, e (iii) para sondas de 30 e $35 \mathrm{~cm}$, a média dos valores de umidade gravimétrica obtidos a 0,$05 ; 0,10 ; 0,20$ e $0,30 \mathrm{~m}$ de profundidade.

\section{Sistema de aquisição de dados com equipamento TDR}

O sistema de aquisição de dados com o equipamento TDR para medição de umidade do solo consiste de um osciloscópio que, por sua vez, converte cada uma das curvas, em 2.000 pares ordenados.

As sondas eram cuidadosamente inseridas no recipiente, isoladas e em posição diferente das anteriores, para evitar interferências. Após a instalação da sonda, faziam-se três leituras consecutivas do pulso refletido, armazenando as informações em planilha eletrônica, procedimento este que gerou o total de 1.656 arquivos.

De posse dos dados dos pulsos refletidos e coletados com o TDR, a próxima etapa foi determinar o tempo de reflexão ocorrido para, depois, se calcular o valor da constante dielétrica do solo. O tempo de reflexão do pulso, ou tempo de trânsito, consiste em determinar os pontos nos quais ocorreram o início e o fim da reflexão. Chamando-se o início e o

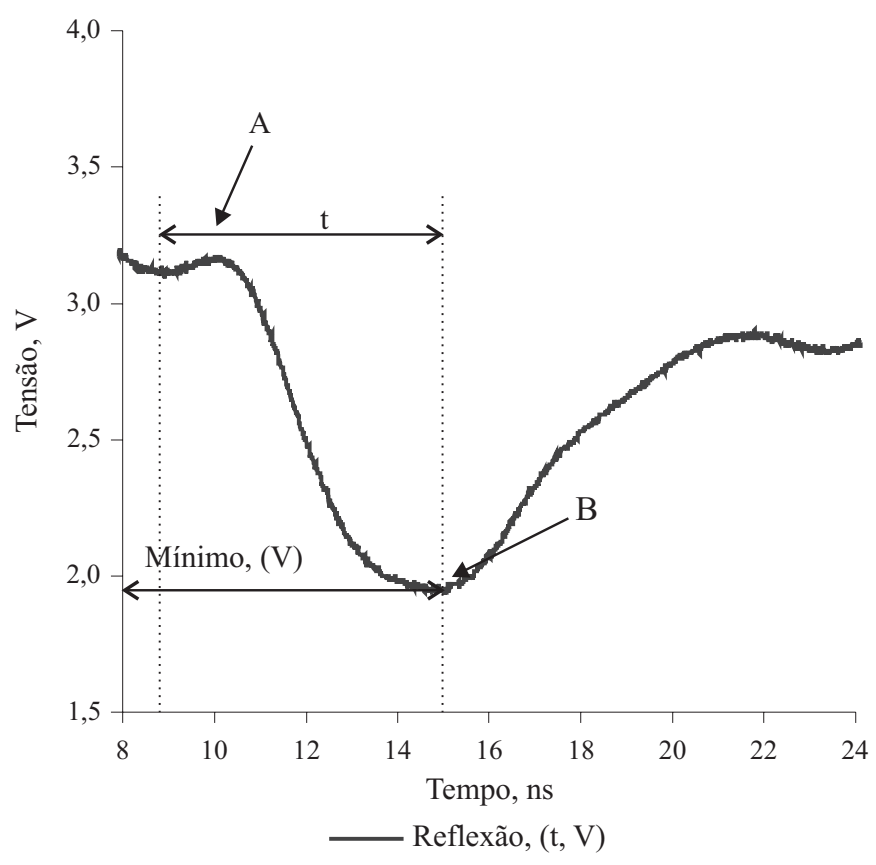

Figura 2. Tempo de trânsito para reflexão no solo Latossolo Vermelho, comprimento de sonda de $0,15 \mathrm{~m}$, diâmetro de 6,5 $\mathrm{mm}$ e umidade média $\theta$ de $0,078 \mathrm{~cm}^{3} \mathrm{~cm}^{-3}$

fim da reflexão como pontos A e B, respectivamente, conforme Figura 2, o tempo de reflexão t será a diferença entre $\mathrm{T}_{\mathrm{A}}$ e $\mathrm{T}_{\mathrm{B}}$.

Um critério essencial para medição da umidade do solo, utilizando-se a tecnologia TDR, é a habilidade em se identificar os pontos A e B, em que o ponto A pode ser determinado como aquele que fica inalterado na curva, sejam quais forem as condições de salinidade e umidade; já o ponto B é correspondente, em tempo, à mínima tensão e pode desaparecer, sob algumas condições (Dalton, 1992; Nadler et al., 1999). Neste estudo, o ponto B, que determina o fim da reflexão, foi definido de acordo com o tempo correspondente ao menor valor de tensão para determinado intervalo, Figura 3. Para cada pulso, este valor foi determinado via software.

O software desenvolvido possui a função de identificar e contar quantas vezes ocorre o valor mínimo de tensão dentro

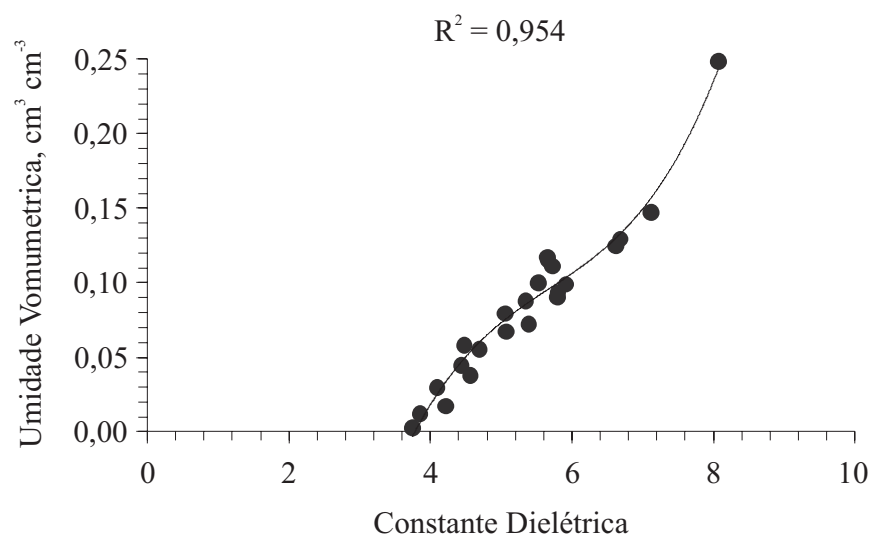

Figura 3. Umidade volumétrica em função da constante dielétrica do solo Areia Quartzosa, para sondas de 0,30 m de comprimento com diâmetros de hastes de $3,2 \mathrm{~mm}$ 
de um intervalo predeterminado. Para se obter o tempo da reflexão $t$, calcula-se a média dos valores correspondentes ao tempo, em ns $\left(10^{-9} \mathrm{~s}\right)$ para a primeira observação do mínimo valor de tensão (ponto B) e para o tempo correspondente à última observação deste. Com o tempo médio entre a primeira e última observação do valor mínimo de tensão e o valor predeterminado do ponto A, foi finalmente calculado, pelo software, o valor do tempo da reflexão t e, a partir deste, a constante dielétrica do solo. O software desenvolvido calcula também os seguintes valores: (i) mínima tensão observada; (ii) número de ocorrências em que este valor é observado, e (iii) tempo de reflexão t. Para simplificação da construção do programa fez-se opção por construí-lo de forma que ele analisasse apenas os dados de um tipo de sonda, para dado diâmetro e em um tipo de solo por vez.

Para se definir o início da reflexão, ou ponto A, calculou-se a máxima variação do tempo de reflexão $\left(\Delta \mathrm{T}_{\max }\right)$, que poderia ocorrer para determinada sonda. $\mathrm{O}$ intervalo de tempo para máxima reflexão teórica foi obtido rearranjando-se a Eq. 3, isto é, calculando-se o tempo de reflexão em função do comprimento da sonda e da constante dielétrica da água. Admitindo-se o valor da constante dielétrica da água $\mathrm{k}_{\mathrm{ag}}$ igual a 80 , o que corresponderia a uma umidade em volume de 1 (ou 100\%), segundo a curva de ajuste de Topp et al. (1980), calculou-se o tempo de reflexão correspondente $\left(\Delta \mathrm{T}_{\text {água }}\right)$; Procedendo-se da mesma forma para o ar e se admitindo $\mathrm{k}_{\mathrm{ar}}$ igual 1 , obteve-se o valor da mínima reflexão $\left(\Delta \mathrm{T}_{\mathrm{ar}}\right)$. A diferença entre os tempos de reflexão para $\mathrm{k}_{\mathrm{ag}}$ igual a 80 e $\mathrm{k}_{\mathrm{ar}}$ igual a 1 , seria o máximo intervalo de reflexão $\left(\Delta \mathrm{T}_{\max }\right)$ que poderia ocorrer para determinada sonda; já para a determinação de valores reais do tempo de trânsito, as sondas foram imersas em água e se obteve a imagem gerada pelo osciloscópio, convertida em pares ordenados e, posteriormente, analisada, repetindo este procedimento para as sondas ao ar. A diferença encontrada dos tempos de reflexão foi considerada o intervalo máximo que poderia ocorrer para aquela sonda $\left(\Delta \mathrm{T}_{\max }\right)$. De posse do valor deste intervalo e do valor do fim da reflexão, quando a sonda se encontra imersa na água $\left(\mathrm{T}_{\mathrm{B} \text { água }}\right.$ ), obtido no eixo do tempo (eixo das abscissas da Figura 3), calculou-se qual seria o valor do ponto A, ou seja, do início da reflexão, por meio da equação

$$
\mathrm{T}_{\mathrm{A}}=\mathrm{T}_{\mathrm{B} \text { água }}-\Delta \mathrm{T}_{\max }
$$

em que

$\mathrm{T}_{\mathrm{A}}$ - início da reflexão, $\mathrm{s}$

$\Delta \mathrm{T}_{\max }$ - intervalo máximo de reflexão, $\mathrm{s}$

$\mathrm{T}_{\mathrm{B} \text { água }}$ - tempo do fim da reflexão para sondas imersas em água, s

\section{Análise estatística}

O modelo de ajuste selecionado para correlação entre a constante dielétrica e a umidade volumétrica do solo, foi do tipo polinomial cúbico. Neste modelo considerou-se variável independente a constante dielétrica do solo e, como dependente, o conteúdo de água no solo, enquanto para o cálculo da constante dielétrica se utilizou a média do tempo de reflexão obtida nas três repetições realizadas com o sistema TDR desenvolvido para cada sonda em cada um dos 23 recipientes. Considerou-se, para o conteúdo de água em volume, a umidade média em volume para cada tipo de solo.

Os pares de dados relativos à constante dielétrica do solo, calculada com o tempo de reflexão t, e a sua umidade volumétrica, para cada tipo de sonda e solo, foram submetidos à análise de variância, pelo teste $\mathrm{F}$, a nível de $1 \%$ probabilidade. Os parâmetros estimados foram testados com a hipótese de nulidade para parâmetro igual a zero, pelo teste t de Student. Determinou-se também o coeficiente de determinação ajustado para as regressões obtidas. O software GENES (Genética Quantitativa e Estatística Experimental, versão 0.1.0) foi utilizado nessas análises.

A fim de se verificar qual o melhor ajuste para o conjunto de dados observados, utilizou-se também o software "CurveExpert”, versão 1.2, programa este que tem a característica de testar diversos modelos de ajuste, indicando o melhor.

\section{RESULTADOS E DISCUSSÃO}

\section{Caracterização dos solos}

A Tabela 1 apresenta a análise granulométrica, a classificação textural dos solos e a caracterização dos solos, para elaboração das suas respectivas curvas de retenção de água.

\section{Impedância das sondas}

Da Tabela 2 tem-se os valores das impedâncias das hastes de $3,2\left(d_{1}\right)$ e $6,5 \mathrm{~mm}\left(\mathrm{~d}_{2}\right)$, em função do teor de umidade do solo e da sua constante dielétrica, para uma separação entre as hastes de $50 \mathrm{~mm}$. Os valores encontrados estão de acordo com Dalton (1992), que demonstrou que o uso de um casador de impedâncias 1:4, nos valores de 50:200 $\Omega$, forneceu resultados satisfatórios para as variações de impedância ocorridas nas hastes metálicas, na condição de solo úmido. O casador de impedância utilizado de 75:300 $\Omega$, mostrou-se componente indispensável para o funcionamento do sistema; no entanto, não foi o mais indicado para todas as umidades. Para a situação de solo saturado, ou quase saturado, ocorreu uma atenuação maior do sinal, impedindo

Tabela 1. Análise granulométrica e classe textural e umidades retidas a diferentes tensões para os solos Areia Quartzosa e Latossolo Vermelho

\begin{tabular}{|c|c|c|c|c|c|c|c|c|c|c|}
\hline \multirow[b]{2}{*}{ Solo } & \multirow[b]{2}{*}{$\begin{array}{l}\text { Areia grossa } \\
\text { dag kg-1 }^{-1}\end{array}$} & \multirow[b]{2}{*}{$\begin{array}{r}\text { Areia fina } \\
\text { dag } \mathrm{kg}^{-1}\end{array}$} & \multirow{2}{*}{$\begin{array}{c}\text { Silte } \\
\text { dag } \mathrm{kg}^{-1}\end{array}$} & \multirow{2}{*}{$\begin{array}{c}\text { Argila } \\
\text { dag } \mathrm{kg}^{-1}\end{array}$} & \multirow[b]{2}{*}{$\begin{array}{l}\text { Classe } \\
\text { textural }\end{array}$} & \multicolumn{5}{|c|}{ Umidade do solo base seca, \% } \\
\hline & & & & & & $\begin{array}{c}\text { Pressão, } \\
\mathrm{MPa}\end{array}$ & 0,03 & 0,1 & 0,5 & 1,5 \\
\hline $\begin{array}{l}\text { Areia } \\
\text { Quartzosa }\end{array}$ & 54,0 & 40,0 & 4,0 & 2,0 & Areia & 17,4 & 9,7 & 6,9 & 4,4 & 3,7 \\
\hline $\begin{array}{l}\text { Latossolo } \\
\text { Vermelho }\end{array}$ & 17,0 & 12,0 & 7,0 & 64,0 & Muito argilosa & 37,3 & 29,7 & 27,0 & 23,9 & 20,4 \\
\hline
\end{tabular}


Tabela 2. Impedâncias correspondentes para diâmetros de hastes de 3,2 $\left(d_{1}\right)$ e $6,5 \mathrm{~mm}\left(d_{2}\right)$, em função do teor de umidade e da constante dielétrica do solo, para uma separação entre as hastes de $0,05 \mathrm{~m}$

\begin{tabular}{cccc}
\hline $\boldsymbol{\theta}$ & ka & $\mathbf{z}\left(\mathbf{d}_{\mathbf{1}}\right)$ & $\mathbf{z}\left(\mathbf{d}_{2}\right)$ \\
$\mathbf{c m}^{\mathbf{3}} \mathbf{c m}^{-3}$ & decimal & $\Omega$ & $\Omega$ \\
0,444 & 30 & 75,4 & 59,9 \\
0,400 & 25 & 82,6 & 65,6 \\
0,345 & 20 & 92,4 & 73,3 \\
0,276 & 15 & 106,6 & 84,7 \\
0,188 & 10 & 130,6 & 103,7 \\
0,080 & 5 & 184,7 & 146,7 \\
0,055 & 4 & 206,5 & 164,0 \\
0,030 & 3 & 238,5 & 189,4 \\
0,003 & 2 & 292,1 & 231,9 \\
\hline
\end{tabular}

sua análise. Acredita-se que casadores de impedância de relação de transformação variáveis devam ser usados para se poder aumentar a sensibilidade dos medidores de umidade do solo usando-se o método TDR.

\section{Homogeneidade da umidade dos solos}

$\mathrm{O}$ método utilizado para se obter a umidade homogênea do solo em todo o perfil, mostrou-se eficiente, uma vez que, ao longo do recipiente, a umidade gravimétrica apresentouse praticamente constante, variando menos de $0,01 \mathrm{~g} \mathrm{~g}^{-1}$ para quase a totalidade dos recipientes para os solos estudados. Obteve-se, também, uma distribuição de umidade uniformemente espaçada entre a umidade residual do solo secado ao ar e de saturação, como desejado.

Os resultados da umidade em volume, tanto quanto aqueles da umidade em peso, tiveram o mesmo padrão, porém a umidade em volume não se distribuiu uniformemente espaçada entre a umidade residual e a saturação do solo. Tal fato é devido aos diferentes valores de densidade úmida, encontrados nos diversos recipientes. No Latossolo Vermelho ocorreu variação entre 1,0 e 1,47 $\mathrm{g} \mathrm{cm}^{-3}$, para o solo secado em estufa e saturado, respectivamente e, na Areia Quartzosa, uma variação de 1,04 a $1,55 \mathrm{~g} \mathrm{~cm}^{-3}$. Acredita-se que esta variação de valores de densidade úmida do solo se deve principalmente à variação nos teores de umidade e à composição e classificação do solo.

\section{Tempo de reflexão}

O tempo máximo de reflexão $\left(\Delta \mathrm{T}_{\max }\right)$ teórica e o tempo máximo de reflexão real encontrados para as doze sondas, são apresentados na Tabela 3. Os limites para o início e o término da reflexão do pulso eletromagnético para os solos Areia Quartzosa e Latossolo Vermelho, encontram-se na Tabela 4. Para as sondas de 0,30 e 0,35 m observou-se, nos dois diâmetros testados, que a atenuação do sinal continuava, após o intervalo determinado (ponto B), em uma segunda reflexão. Para os referidos comprimentos de sonda, ocorriam reflexões sucessivas quando os solos se encontravam saturados, considerando-se para análise apenas a primeira reflexão. Para contornar este fenômeno, adotou-se como limite máximo para o tempo de reflexão, ou ponto $\mathrm{B}$, o valor de 20,5 ns.
Tabela 3. Máximo tempo de reflexão do pulso eletromagnético teórico e real para sondas com diâmetro $\mathrm{d}_{1}$ de $3,2 \mathrm{~mm}$ e $\mathrm{d}_{2}$ de $6,5 \mathrm{~mm}$, em função do comprimento da sonda

\begin{tabular}{cccc}
\hline $\mathbf{L}(\mathbf{m})$ & $\boldsymbol{\Delta} \mathbf{T}_{\max }$ teórico (ns) & $\boldsymbol{\Delta} \mathbf{T}_{\max }$ real $\mathbf{d}_{\mathbf{1}}(\mathbf{n s})$ & $\boldsymbol{\Delta} \mathbf{T}_{\max }$ real $\mathbf{d}_{\mathbf{2}}(\mathbf{n s})$ \\
0,10 & 5,30 & 4,72 & 4,64 \\
0,15 & 7,94 & 5,30 & 5,82 \\
0,20 & 10,59 & 9,93 & 7,97 \\
0,25 & 13,24 & 12,66 & 12,60 \\
0,30 & 15,89 & 13,36 & 13,74 \\
0,35 & 18,54 & 14,98 & 15,39 \\
\hline
\end{tabular}

Tabela 4. Limites para o início $\left(T_{A}\right)$ e término $\left(T_{B}\right)$ da reflexão do pulso eletromagnético, para os solos Areia Quartzosa e Latossolo Vermelho

\begin{tabular}{|c|c|c|c|c|c|c|c|c|}
\hline \multirow{4}{*}{$\begin{array}{l}\text { Com. } \\
\text { da } \\
\text { sonda } \\
\text { L, (m) }\end{array}$} & \multicolumn{4}{|c|}{ Areia Quartzosa, $d_{1}$} & \multicolumn{4}{|c|}{ Latossolo Vermelho, $d_{1}$} \\
\hline & \multirow{2}{*}{\multicolumn{2}{|c|}{$\begin{array}{c}\mathrm{T}_{\mathrm{A}}(\mathrm{ns}) \\
\text { Diâmetro da } \\
\text { sonda }(\mathrm{mm})\end{array}$}} & \multirow{2}{*}{\multicolumn{2}{|c|}{$\begin{array}{c}\mathrm{T}_{\mathrm{B}}(\mathrm{ns}) \\
\text { Diâmetro da } \\
\text { sonda }(\mathrm{mm})\end{array}$}} & \multirow{2}{*}{\multicolumn{2}{|c|}{$\begin{array}{c}\mathrm{T}_{\mathrm{A}}(\mathrm{ns}) \\
\text { Diâmetro da } \\
\text { sonda }(\mathrm{mm})\end{array}$}} & \multirow{2}{*}{\multicolumn{2}{|c|}{$\begin{array}{c}\mathrm{T}_{\mathrm{B}}(\mathrm{ns}) \\
\text { Diâmetro da } \\
\text { sonda }(\mathrm{mm})\end{array}$}} \\
\hline & & & & & & & & \\
\hline & 3,2 & 6,5 & 3,2 & 6,5 & 3,2 & 6,5 & 3,2 & 6,5 \\
\hline 0,10 & 10,50 & 10,50 & 16,57 & 16,60 & 10,50 & 10,50 & 16,57 & \\
\hline 0,15 & 10,50 & 10,50 & 17,81 & 18,09 & 10,50 & 10,50 & 17,81 & 18,09 \\
\hline 0,20 & 10,20 & 10,20 & 21,41 & 17,93 & 10,50 & 10,20 & 21,41 & 17,93 \\
\hline 0,25 & 10,60 & 10,60 & 15,50 & 25,93 & 10,50 & 10,60 & 15,50 & 25,93 \\
\hline 0,30 & 10,30 & 10,30 & 27,11 & 27,21 & 9,50 & 10,30 & 27,11 & 27,21 \\
\hline 0,35 & 10,10 & 10,10 & 28,89 & 28,95 & 9,00 & 10,10 & 28,89 & 28,95 \\
\hline
\end{tabular}

Constatou-se que o ponto tensão mínima (ponto B), dentro do intervalo selecionado entre os limites predeterminados por $T_{A}$ e $T_{B}$, poderia repetir-se várias vezes. $A$ conversão da imagem visualizada no osciloscópio em 2.000 pares ordenados aumenta, em muito, a resolução da leitura do tempo (0,01 ns) e, por este motivo, podem ocorrer vários pontos (pares ordenados) para uma mesma tensão mínima.

\section{Relação entre constante dielétrica e umidade volumétrica do solo}

De posse dos valores da umidade volumétrica e constante dielétrica do solo, obteve-se a curva de calibração para os diversos tipos de sonda, nos dois solos estudados.

O modelo polinomial cúbico ajustou-se bem à calibração do sistema. A Figura 3 apresenta a umidade volumétrica em função da constante dielétrica aparente para o solo Areia Quartzosa, para sonda de 0,30 m e de haste de 3,2 mm. O coeficiente de correlação encontrado foi de 0,954 .

A dispersão em torno das curvas ajustadas pode ter ocorrido em razão de características não uniformes do solo, como densidade, compactação ou porosidade. A variação da densidade úmida de 1,0 a 1,47 para o solo Latossolo Vermelho e de 1,04 a 1,55 para o solo Areia Quartzosa, revela-se como sendo a maior responsável pela dispersão ocorrida em torno das curvas ajustadas. Uma vez que os dois tipos de solo estudado foram depositados uniformemente dentro dos recipientes, infere-se que a compactação tende a ser uniforme para os diversos recipientes, embora este aspecto não tenha sido avaliado; da mesma forma, diz-se para a porosidade do solo. 


\section{Análise estatística}

A análise dos dados indicou que todos os modelos encontrados apresentaram níveis de significância, pelo teste F, superiores a 0,99 de probabilidade. A Tabela 5 apresenta o resumo das análises de variância das regressões, contendo o coeficiente de determinação ajustado, os parâmetros estimados e os valores dos testes t das estimativas dos parâmetros, para os solos Areia Quartzosa e Latossolo Vermelho, com diâmetros de hastes de 3,2 e 6,5 mm, respectivamente. Essa tabela apresenta também os resultados encontrados para uma curva geral, com todos os comprimentos de sonda, de um diâmetro para um solo, denominados $\mathrm{Td}_{1 \mathrm{AQ}}, \mathrm{Td}_{2 \mathrm{AQ}}, \mathrm{Td}_{1 \mathrm{LV}}$ e $\mathrm{Td}_{2 \mathrm{LV}}$.

Para o solo Areia Quartzosa e sondas com diâmetro de haste de 3,2 mm, verifica-se que o melhor ajuste ocorreu para a sonda com $0,30 \mathrm{~m}$ de comprimento de haste, sonda de $0,35 \mathrm{~m}$. Para este conjunto de sondas, os testes de hipóteses das estimativas dos parâmetros sugerem que os termos independentes, os coeficiente lineares, quadráticos e cúbicos, têm probabilidade menor que 1,9\% de serem nulos, ou seja, de não afetarem média da resposta para o termo dependente.

Para o solo Areia Quartzosa e sondas com diâmetro de hastes de 6,5 mm, o melhor ajuste se deu com a sonda de 0,20 m, seguindo-se a sonda de 0,35 m. Para este conjunto de sondas os testes de hipóteses das estimativas dos parâmetros sugerem que o coeficiente cúbico apresenta, para a sonda de $0,10 \mathrm{~m}$, probabilidade maior que $62,7 \%$ de ser nulo, isto é, de não afetar a resposta para a variável dependente, indicando que o modelo polinomial cúbico pode não ser o melhor ajuste para os dados observados.

Para o solo Latossolo Vermelho e sondas com diâmetro de hastes de 3,2 mm, verifica-se que o melhor ajuste ocorreu com a sonda de comprimento $0,35 \mathrm{~m}$, seguindo-se a sonda de $0,15 \mathrm{~m}$. Para este conjunto de sondas os testes de hipóteses das estimativas dos parâmetros indicam que os coeficientes quadráticos, para sondas de 0,20 e $0,25 \mathrm{~m}$ e o coeficiente cúbico, para a sonda de $0,30 \mathrm{~m}$, têm probabilidades maiores que 79,6 , 97,5 e 77,2\% de serem nulos, respectivamente, cujos eventos levam ao questionamento sobre o uso do modelo polinomial cúbico para relacionar a constante dielétrica com a umidade volumétrica.

Para o solo Latossolo Vermelho e sondas com diâmetro de hastes de $6,5 \mathrm{~mm}$, vê-se que o melhor ajuste ocorreu para a sonda de 0,10 m, seguindo-se a sonda de 0,35 m. Para este conjunto de sondas, os testes de hipótese das estimativas dos

Tabela 5. Análise de variância das regressões entre a constante dielétrica e a umidade volumétrica, para areia Quartzosa e Latossolo Vermelho

\begin{tabular}{|c|c|c|c|c|c|c|c|c|c|}
\hline \multirow{2}{*}{$\begin{array}{l}\text { Comprimento } \\
\text { da sonda, }(m)\end{array}$} & \multirow{2}{*}{$R^{2}$ aj } & \multirow{2}{*}{ A } & \multirow{2}{*}{ ka } & \multirow{2}{*}{$\mathrm{ka}^{2}$} & \multirow{2}{*}{$\mathrm{Ka}^{3}$} & \multicolumn{4}{|c|}{ Prob $>\mathrm{T}$} \\
\hline & & & & & & a & $\mathrm{Ka}$ & $\mathbf{k a}^{2}$ & $\mathbf{k a}^{3}$ \\
\hline \multicolumn{10}{|c|}{ Areia Quartzosa e sondas com 3,2 mm de diâmetro } \\
\hline 0,10 & 0,885 & $-0,8995$ & 0,2677 & $-0,02456$ & 0,000771 & 0,0004 & 0,0004 & 0,0008 & 0,0008 \\
\hline 0,15 & 0,899 & $-0,5263$ & 0,1804 & $-0,01773$ & 0,000626 & 0,0046 & 0,0065 & 0,0165 & 0,0190 \\
\hline 0,20 & 0,892 & $-0,5226$ & 0,1687 & $-0,01551$ & 0,000511 & 0,0019 & 0,0036 & 0,0129 & 0,0187 \\
\hline 0,25 & 0,927 & $-0,7767$ & 0,4189 & $-0,07024$ & 0,004146 & 0,0014 & 0,0020 & 0,0035 & 0,0032 \\
\hline 0,30 & 0,954 & $-1,0654$ & 0,5509 & $-0,09146$ & 0,005365 & 0,0009 & 0,0014 & 0,0022 & 0,0017 \\
\hline 0,35 & 0,946 & $-1,6703$ & 0,8916 & $-0,15412$ & 0,009154 & 0,0002 & 0,0002 & 0,0004 & 0,0003 \\
\hline $\mathrm{Td}_{1 \mathrm{AQ}}$ & 0,455 & $-0,2838$ & 0,1224 & $-0,01305$ & 0,000474 & 0,0002 & 0,0000 & 0,0003 & 0,0004 \\
\hline \multicolumn{10}{|c|}{ Areia Quartzosa e sondas com 6,5 mm de diâmetro } \\
\hline 0,10 & 0,867 & $-0,2603$ & 0,05029 & $-0,002322$ & 0,0000446 & 0,0900 & 0,1360 & 0,3196 & 0,6270 \\
\hline 0,15 & 0,916 & $-0,3014$ & 0,08727 & $-0,006830$ & 0,0002156 & 0,0141 & 0,0251 & 0,0788 & 0,0859 \\
\hline 0,20 & 0,924 & $-0,6907$ & 0,21272 & $-0,020157$ & 0,0006917 & 0,0037 & 0,0065 & 0,0142 & 0,0148 \\
\hline 0,25 & 0,895 & $-0,7249$ & 0,34192 & $-0,050876$ & 0,0027353 & 0,0263 & 0,0355 & 0,0517 & 0,0466 \\
\hline 0,30 & 0,898 & $-1,2199$ & 0,58899 & $-0,092251$ & 0,0050965 & 0,0289 & 0,0397 & 0,0542 & 0,0526 \\
\hline 0,35 & 0,920 & $-1,4412$ & 0,67971 & $-0,102002$ & 0,0052790 & 0,0006 & 0,0011 & 0,0021 & 0,0024 \\
\hline $\mathrm{Td}_{2 \mathrm{AQ}}$ & 0,314 & $-0,0892$ & 0,04042 & $-0,002741$ & 0,0000675 & 0,0599 & 0,0053 & 0,0291 & 0,0351 \\
\hline \multicolumn{10}{|c|}{ Latossolo Vermelho e sondas com 3,2 mm diâmetro } \\
\hline 0,10 & 0,878 & $-0,1046$ & 0,02119 & $-0,000446$ & 0,00000365 & 0,0446 & 0,0007 & 0,0133 & 0,0296 \\
\hline 0,15 & 0,895 & $-0,1205$ & 0,03105 & $-0,000945$ & 0,00001511 & 0,0780 & 0,0244 & 0,2486 & 0,3312 \\
\hline 0,20 & 0,865 & $-0,0829$ & 0,02340 & 0,000386 & $-0,0000219$ & 0,2869 & 0,2317 & 0,7967 & 0,5597 \\
\hline 0,25 & 0,874 & $-0,1359$ & 0,04084 & $-0,000050$ & $-0,0000287$ & 0,0893 & 0,0617 & 0,9754 & 0,5173 \\
\hline 0,30 & 0,888 & $-0,3158$ & 0,07033 & $-0,002064$ & 0,00001654 & 0,0260 & 0,0403 & 0,5800 & 0,7724 \\
\hline 0,35 & 0,919 & $-0,4677$ & 0,10950 & $-0,005154$ & 0,00009261 & 0,0098 & 0,0174 & 0,1484 & 0,3030 \\
\hline $\mathrm{Td}_{1 \mathrm{LV}}$ & 0,549 & $-0,1013$ & 0,03763 & $-0,001073$ & 0,00000947 & 0,0020 & 0,0000 & 0,0000 & 0,0000 \\
\hline \multicolumn{10}{|c|}{ Latossolo Vermelho e sondas com 6,5 mm de diâmetro } \\
\hline 0,10 & 0,923 & $-0,03837$ & 0,01115 & $-0,000149$ & 0,0000015 & 0,3027 & 0,0179 & 0,6369 & 0,6029 \\
\hline 0,15 & 0,906 & 0,01123 & $-0,01039$ & 0,001769 & $-0,0000313$ & 0,8200 & 0,5299 & 0,1220 & 0,2263 \\
\hline 0,20 & 0,870 & $-0,02728$ & $-0,00295$ & 0,002434 & $-0,0000646$ & 0,7487 & 0,8768 & 0,1058 & 0,0588 \\
\hline 0,25 & 0,850 & $-0,45005$ & 0,11076 & $-0,005057$ & 0,0000812 & 0,0141 & 0,0192 & 0,1745 & 0,6064 \\
\hline 0,30 & 0,888 & $-0,29931$ & 0,05915 & $-0,001101$ & $-0,0000054$ & 0,0519 & 0,1043 & 0,6866 & 0,9295 \\
\hline 0,35 & 0,911 & $-0,57953$ & 0,11203 & $-0,004697$ & 0,0000753 & 0,0270 & 0,0611 & 0,2827 & 0,5330 \\
\hline $\mathrm{Td}_{2 \mathrm{LV}}$ & 0,490 & $-0,13639$ & 0,04212 & $-0,001330$ & 0,0000134 & 0,0009 & 0,0000 & 0,0000 & 0,0000 \\
\hline
\end{tabular}


parâmetros sugerem que o termo independente e o coeficiente linear para sonda de 0,20 (maiores que 74,8 e 87,6\%) e coeficientes quadrático e cúbico, para as sondas de 0,10 (maiores que 63,6 e 60,2\%) e 0,30 m (maiores que 68,6 e 92,9\%), e o coeficiente cúbico, para a sonda de 0,25 m (maiores que $60,6 \%$ ) sejam nulos, respectivamente.

Para todas as sondas e solos estudados, os modelos gerais mostraram-se ineficientes, isto é, coeficientes de determinação menores que 55\%, resultando que, para a determinação da variável dependente, ou seja, da umidade do solo, é preferível utilizar-se o modelo específico para cada sonda, em cada tipo de solo.

As sondas de diferentes diâmetros apresentaram coeficientes de determinação similares.

Quanto ao solo Areia Quartzosa, o programa CurveExpert 1.21 apresentou o modelo polinomial cúbico como o de melhor ajuste, quando se correlacionaram dados oriundos de uma sonda isoladamente; enfim, para o modelo geral, com todas as sondas de diâmetro de 3,2 mm $\left(\mathrm{Td}_{1 \mathrm{AQ}}\right)$, o melhor ajuste foi obtido com o modelo polinomial cúbico.

Há necessidade de estudos complementares a fim de aperfeiçoar o sistema TDR proposto para determinação da umidade do solo. Sugere-se que se investigue: (i) quanto ao casador de impedância: analisar a viabilidade de se utilizarem casadores de impedância específicos, por faixa de umidade e (ii) quanto à calibração do sistema TDR proposto, utilizar modelos físicos de calibração que levem em consideração características do solo, como textura, densidade e porosidade.

\section{CONCLUSÕES}

1. O uso de casador de impedâncias 1:4 (75:300 $\Omega$ ) mostrou-se indispensável na maioria dos casos, para o sistema TDR desenvolvido para a medição da umidade do solo.

2. O modelo polinomial cúbico apresentou-se como o melhor ajuste para o solo de textura mais grossa Areia Quartizoza.

3. O método utilizado para a determinação do fim da reflexão do pulso eletromagnético nas sondas, mostrou-se válido para o cálculo da constante dielétrica $(\mathrm{ka})$ do solo.

4. Os melhores ajustes ocorreram para as hastes de 3,2 mm de diâmetro e comprimento de 0,30 m no solo Areia Quartizoza; já para o Latossolo Vermelho, a sonda de melhor ajuste foi aquela com diâmetro de hastes de $6,5 \mathrm{~mm}$ e comprimento de $0,10 \mathrm{~m}$, e nestas condições em que o sistema desenvolvido apresentou maior precisão na determinação do conteúdo de água do solo.

5. Sondas com diferentes diâmetros apresentaram comportamentos similares, quando se compararam apenas os coeficientes de determinação.

6. O sistema TDR desenvolvido foi válido tecnicamente, para determinação da umidade dos solos de textura arenosa e argilosa.

\section{AGRADECIMENTOS}

Os autores são gratos à Fundação de Amparo à Pesquisa, do Estado de Minas Gerais, e à Universidade Federal de Viçosa, pelo apoio para a realização deste trabalho.

\section{LITERATURA CITADA}

Amato, M.; Ritchie, J. T. Small spatial scale soil water content measurement with time-domain reflectometry. Soil Science Society of America Journal, Madison, v.59, p.325329, 1995.

Dalton, F. N. Development of time-domain reflectometry for measuring soil water content and bulk soil electrical conductivity. Soil Science Society of America, Madison, (Advances in measurement of soil physical properties: bringing theory into practice), Special Publication n.30, p.143-167, 1992.

Dirksen, C.; Dasberg, S. Improved calibration of time domain reflectometry soil water content measurements. Soil Science Society of America Journal, Madison, v.57, p.660667, 1993.

Gomide, R. L. Monitoramento para manejo da irrigação: instrumentação, automação e métodos. In: Manejo de irrigação, editado por Faria, M. A. Lavras: Suprema Gráfica, 1998. cap. 2, p.133-280.

Huisman, J. A.; Bouten, W. Comparison of calibration and direct measurement of cable and probe properties in time domain reflectometry. Soil Science Society of America Journal, Madison, v.63 n.6, p.1615-1617, 1999.

Nadler, A.; Gamliel, A.; Peretz, I. Practical aspects of salinity effect on TDR-measured water content: a field study. Soil Science Society of America Journal, Madison, v.63, n.5, p.10701076, 1999.

Nissen, H. H.; Moldrup, P.; Henriksen, K. High-resolution time domain reflectometry coil probe for measuring soil water content. Soil Science Society of America Journal, Madison, v.62, n.5, p.1203-1211, 1998.

Noborio, K.; Horton, R.; Tan, C. S. Time domain reflectometry probe for simultaneous measurement of soil matric potential and water content. Soil Science Society of America Journal, Madison, v.63, n.6, p.1500-1505, 1999.

Robinson, D. A.; Bell, J. P.; Batchelor, C. H. Influence of iron and titanium on water content determination by TDR. Symposium: Time Domain Reflectometry Applications in Soil Science held at the Research Centre Foulum, 1994, Denmark. Proceedings.... Denmark: Danish Institute of Plant and Soil Science, 1994, p.63-70.

Roth, C. H.; Malicki, M. A.; Plagge, R. Empirical evaluation of the relationship between soil dielectric constant and volumetric water content as the basis for calibrating soil moisture measurements by TDR. Journal of Soil Science, Reading, v.43, p.1-13, 1992. 
Serrarens, D.; Macintyre, J. L.; Hopmans, J. W.; Bassoi, L. H. Soil moisture calibration of TDR multilevel probes. Scientia Agricola, Piracicaba, v.57, n.2, p.349-354, 2000.

Spaans, E. J. A.; Baker, J. M. Simple baluns in parallel probes for time domain reflectometry. Soil Science Society of America Journal, Madison, v.3, n.57, p.668-673, 1993.

Tommaselli, J. T. G. Influência de algumas características do solo sobre a calibração de um aparelho de TDR (time domain reflectometry). Piracicaba: Centro de Energia Nuclear na Agricultura, 1997. 109p.Tese Doutorado
Topp, G. C.; Davis, J. L.; Annan, A. P. Electromagnetic determination of soil water content: measurements in coaxial transmission lines. Water Resources Research, Las Cruces, v.16, n.3, p.574-582, 1980.

White, I.; Zegelin, S. J.; Topp, G. C.; Fish, A. Effect of bulk electrical conductivity on TDR measurement of water content in porous media. Symposium and Workshop on Time Domain Reflectometry in Environmental, Infrastructure, and Mining Applications, 1994, Evanston. Proceedings.... Evanston: Northwestern University Illinois. 1994. p.294-308. 